\title{
Effect of nuclear quadrupole interactions on the dynamics of two-level systems in glasses
}

\author{
A. L. Burin ${ }^{\dagger}$, I.Ya.Polishchuk ${ }^{\ddagger}$, P. Fulde ${ }^{\star}$, Y. Sereda ${ }^{\dagger}$ \\ ${ }^{\dagger}$ Department of Chemistry, Tulane University, New Orleans, LA 70118 \\ ${ }^{\ddagger} R R C$ Kurchatov Institute, Kurchatov Sq. 1, 123182 Moscow, Russia and \\ - Max-Planck-Institut für Physik Komplexer Systeme, D-01187 Dresden, Germany
}

(Dated: November 20, 2018)

\begin{abstract}
The standard tunneling model describes quite satisfactorily the thermal properties of amorphous solids at temperatures $T<1 K$ in terms of an ensemble of two-level systems possessing logarithmically uniform distribution over their tunneling amplitudes and uniform distribution over their asymmetry energies. In particular, this distribution explains the observable logarithmic temperature dependence of the dielectric constant. Yet, experiments have shown that at ultralow temperatures $T<5 m K$ such a temperature behavior breaks down and the dielectric constant becomes temperature independent (plateau effect). In this letter we suggest an explanation of this behavior exploiting the effect of the nuclear quadrupole interaction on tunneling. We show that below a temperature corresponding to the characteristic energy of the nuclear quadrupole interaction the effective tunneling amplitude is reduced by a small overlap factor of the nuclear quadrupole ground states in the left and right potential wells of the tunneling system. It is just this reduction that explains the plateau effect . We predict that the application of a sufficiently large magnetic field $B>10 T$ should restore the logarithmic dependence because of the suppression of the nuclear quadrupole interaction.
\end{abstract}

PACS numbers: 6143.Fs, 77.22.Ch,75.50Lk

Low temperature properties of amorphous solids have been associated with two-level systems (TLS) 1, 2]. Each TLS can be described by the tunneling amplitude $\Delta_{0}$ and the two-well asymmetry energy $\Delta$. The tunneling amplitude $\Delta_{0}$ couples two energy minima and it is exponentially sensitive to external and internal parameters of the system. This results in the logarithmically uniform distribution [1, 2, 3, 4]

$$
P\left(\Delta, \Delta_{0}\right)=\frac{P}{\Delta_{0}}, P=\text { const. }
$$

Equation (1) leads to a universal temperature and time dependence of various physical properties of amorphous solids, thus making "glassy" behavior easily recognizable. One of the signatures of the glassy state is a logarithmic temperature dependence of the dielectric constant and the sound velocity [3, 4].

Recent experimental investigations on many amorphous solids at very low temperature have revealed a number of qualitative deviations from the standard tunneling model. In particular, it was demonstrated [5, 6, 7, 8] that for $T \leq 5 m K$ the dielectric constant is approximately temperature independent rather than logarithmically temperature dependent. These results conflict with the logarithmically uniform distribution Eq. (11) of TLS over tunneling amplitudes. To resolve the problem one can assume that the distribution of TLS has a low energy cut-off at $\Delta_{0, \min } \sim 1-3 m K$ (See [ 5 ], Fig. 11. Although this assumption fits the experimental data quite good, it is in conflict with the observation of very long relaxation times in glasses 9 . These times (a week or longer) require much smaller tunneling amplitudes than $1 m K$ (remember that the TLS relaxation time is inversely proportional to its squared tunneling amplitude).

We suggest an explanation for this controversial behavior using recent work 10, 11, 12], where it was proposed that the nuclear quadrupole interaction affects the properties of TLS's at low temperatures due to a mismatch of the nuclear quadrupole states in the two potential wells 13. The case of higher temperatures $T>5 m K$ has been considered in 11, 12]. The significance of the nuclear quadrupole interaction has been proven experimentally in glycerol glass [14]. This interaction also helps to understand the unusual magnetic field dependence of dielectric properties in non-magnetic dielectric glasses [7, 8].

The nuclear quadrupole interaction splits the levels of a well and the effective tunneling between pairs of them is $\Delta_{0 *}<\Delta_{0}$, i.e., gets smaller than the initial value $\Delta_{0}$. We demonstrate that this reduction is of decisive importance for TLS with tunneling amplitude $\Delta_{0}$ smaller than the characteristic nuclear quadrupole interaction energy $\lambda_{*}$. The contribution of these TLS's to the dielectric constant is substantially suppressed. This can explain the experimental observations.

First, we review the key data concerning the contribution of tunneling systems to the resonant part of the dielectric susceptibility for $T<50 \mathrm{mK}[3$, 4]. This contribution can be described as the adiabatic response of the quantum system in the ground state to a slow external field. The application of an external electric field to the tunneling system in the ground state produces a polarization along the field. For instance, in the twolevel model the external field $\mathbf{E}$ affects the TLS Hamiltonian through the change in the asymmetry energy, i.e., $-\Delta_{0} s^{x}-(\Delta-\boldsymbol{\mu} \mathbf{E}) s^{z}[3,4]$. The ground state has the 
energy $E_{g}=-(1 / 2) \sqrt{\Delta^{2}+\Delta_{0}^{2}}$, which results in the susceptibility $\chi_{g}=-\frac{\mu^{2}}{3} d^{2} E_{g} / d \Delta^{2}=\frac{\mu^{2}}{3} \frac{\Delta_{0}^{2}}{2\left(\Delta_{0}^{2}+\Delta^{2}\right)^{3 / 2}}$.

A contribution of excited states to the susceptibility can be negative. In a two-level system the contribution of the excited state with the energy $E_{e}=(1 / 2) \sqrt{\Delta^{2}+\Delta_{0}^{2}}$ is $-\chi_{g}$. When the temperature is less than the minimum energy splitting between the ground and excited state $E_{\min }\left(\Delta_{0}\right)=\Delta_{0}$, the excited state contribution can be neglected. Then, the contribution of the TLS's with given $\Delta_{0}>T$ and different $\Delta$ to the susceptibility is

$$
-P \frac{\mu^{2}}{3} \int_{-W}^{W} d \Delta \frac{d^{2} E_{g}}{d \Delta^{2}}=-\left.P \frac{\mu^{2}}{3} \frac{d E_{g}\left(\Delta_{0}, \Delta\right)}{d \Delta}\right|_{-W} ^{W},
$$

where $W \gg \Delta_{0}, T$ is the maximum TLS asymmetry. For large enough $\Delta$ one has $E_{g}(\Delta) \sim-|\Delta| / 2$ and the integral (2) equals unity. This result is valid for the ground state of the tunneling system irrespective of the presence of the nuclear quadrupole interaction. Then, one should make averaging of $P \frac{\mu^{2}}{3}$ over $\Delta_{0}$ using the distribution Eq. (11) with the constraint that the minimum energy splitting $E_{\min }$ between the ground- and first excited state exceeds $T$. This is realized when $\Delta_{0}>T$. Otherwise, the positive dielectric susceptibility of the ground state is compensated by the negative contribution of the excited state and the total contribution can be neglected. Then one may express the TLS resonant susceptibility to logarithmic accuracy in the form

$$
\chi_{T L S} \approx \frac{P \mu^{2}}{3} \int_{0}^{W} \frac{d \Delta_{0}}{\Delta_{0}} \Theta\left(E_{\min }\left(\Delta_{0}\right)-T\right),
$$

where $\Theta(x)$ is the Heaviside step function. In the standard TLS model $E_{\text {min }}\left(\Delta_{0}\right)=\Delta_{0}, 3$, 4] and we obtain the well-known result $\chi_{T L S} \approx\left(P \mu^{2} / 3\right) \ln (W / T)$. Equation (3) is still applicable in the low temperature limit, where the nuclear quadrupole interaction is significant.

To describe the effect of the nuclear quadrupole interaction on the dielectric constant, we consider the change in TLS ground state properties induced by this interaction. The adiabatic (resonant) dielectric susceptibility $\chi \propto \Delta^{-3}$ (see Refs. [3, 4] ) is due to the TLS's with small asymmetry energies $\Delta \leq \Delta_{0}$. Moreover, as discussed previously, the minimum energy splitting between the ground and first excited state takes place at $\Delta=0$. Therefore, in what follows we study this particular case.

Consider a two-level system formed by $n$ tunneling atoms possessing a nuclear spin $I \geq 1$ and consequently a nuclear quadrupole moment. The tunneling system Hamiltonian can be expressed through the standard TLS pseudospin Hamiltonian $-\Delta_{0} s^{x}$ and the quadrupole interactions, which are $\widehat{H}_{r}$ and $\widehat{H}_{l}$ in the left well $\left(s^{z}=\right.$ $-1 / 2)$ and the right well $\left(s^{z}=1 / 2\right)$, respectively. The mismatch of the nuclear quadrupole interactions between the two wells makes the TLS properties sensitive to an external magnetic field [10. We also assume that the choice of $\Delta=0$ implies identical ground state energies of nuclear spins in the right and left wells, i.e., $<g l\left|\widehat{H}_{l}\right| g l>=<g r\left|\widehat{H}_{r}\right| g r>=E_{\text {quadr }}^{\text {loc }}$, where $\mid g l>(\mid g r>)$ is the ground nuclear spin state in the left (right) well. The modified TLS Hamiltonian takes the form

$$
\widehat{h}_{T L S}=-\Delta_{0} s^{x}+\left(\widehat{H}_{r}+\widehat{H}_{l}\right) / 2+\left(\widehat{H}_{r}-\widehat{H}_{l}\right) s^{z} .
$$

Two different realizations of the ground state are possible for the Hamiltonian Eq. (4) depending on the relative size of the TLS tunneling amplitude $\Delta_{0}$ and the nuclear quadrupole interaction energy $\lambda_{*}$. If $\Delta_{0}>\lambda_{*}$, then the hybridization is strong and the tunneling particle is equally shared between both wells and one can set $s^{x}=1 / 2, s^{z} \approx 0$ in Eq. (4). Then, the ground state energy is estimated as $E_{g}^{h y b}=-\Delta_{0} / 2+E_{\text {quadr }}^{\text {hyb }}$, where $E_{\text {quadr }}^{\text {hyb }}$ is the ground state energy of the nuclear spin in the hybridized Hamiltonian $\left(\widehat{H}_{r}+\widehat{H}_{l}\right) / 2$. If $\Delta_{0}<\lambda_{*}$, the ground state of the system corresponds to either the localized left well ground state or the right one $E_{\text {quadr }}^{\text {loc }}=<g l\left|\widehat{H}_{l}\right| g l>=<g r\left|\widehat{H}_{r}\right| g r>$. The tunneling term in Eq. (4) leads to a small correction to this energy given by

$$
\delta E=-\eta_{*} \Delta_{0} / 2, \quad \eta_{*}=<g l \mid g r>.
$$

The parameter $\eta_{*}<1$ characterizes the average overlap integral of the nuclear spin ground states in the left and right wells. We assume that $\eta_{*} \ll 1$. For this reason, correction (5) is ignored below when estimating the energy.

The energy splitting between the ground and the first excited state, formed by the symmetric and antisymmetric superpositions of the localized ground states in the left and right wells is given by the modified effective tunneling amplitude

$$
\Delta_{0 *}=\eta_{*} \Delta_{0}=E_{\min }\left(\Delta_{0}\right),
$$

which also defines the minimum energy splitting of the ground and first excited states $E_{\min }\left(\Delta_{0}\right)$, because other excited states can be neglected (see below Eq. (8) and the related discussion).

We have found the two possible realizations for the ground state of TLS's affected by the nuclear quadrupole interaction, a hybridized and a localized one. For the parameter $\lambda_{*}$ introduced above one has

$$
\lambda_{*}=2\left(E_{\text {quadr }}^{\text {hyb }}-E_{\text {quadr }}^{\text {loc }}\right) .
$$

In the case $\Delta_{0}>\lambda_{*}$, i.e., the TLS is hybridized between the left and right wells and the nuclear quadrupole interaction can be approximately ignored. In the opposite case $\Delta_{0}<\lambda_{*}$, the nuclear quadrupole interaction localizes the tunneling particle in one of the wells leading to a strong reduction of the effective tunneling amplitude and replacing $\Delta_{0}$ by $\Delta_{0 *}=\eta_{*} \Delta_{0} \ll \Delta_{0}$. The nuclear 
spin ground state in each well can be treated as nondegenerate 15. Then, one can approximately define the ground state and the lowest excited state as superpositions of these two lowest energy states. The ground state of the nuclear spin in each of the two wells can be considered as non-degenerate and one may construct the ground state and the lowest excited state as a superposition of unperturbed ground states in the two wells. Higher excited state contributions are neglected. This is justified by calculating their contribution to lowest order perturbation theory. We find a correction factor $c$ to the ground state amplitude as

$$
c=1-\sum_{i \neq g r} \frac{\Delta_{0}^{2}|<g l| i>\left.\right|^{2}}{\left(E_{i}-E_{g l}\right)^{2}} .
$$

We determine the parameter regime for which the second term can be neglected as follows. Assume that each TLS contains $n$ simultaneously tunneling atoms. The lowest excited states in each well are separated from the ground state by some characteristic energy $b \sim \hbar \omega_{0}$ where $\omega_{0}$ is a frequency of the nuclear quadrupole resonance. The next group of states is separated by the energy interval $\sim 2 b$. The vast majority of the states have energies exceeding that for the ground state by the energy $n b \sim \lambda_{*} / 2$ (see Eq. (7) and 16]). Because of the large statistical weight, these states provide the main contribution to Eq. (8). So, we may replace the denominator in Eq. (8) by $\lambda_{*}$. Then, the sum of the $\mid\left\langle g l|i>|^{2}\right.$ in the numerator can be approximated by 1 . The requirement that the second term in Eq. (8) is small results in the condition

$$
\Delta_{0}<\lambda_{*} .
$$

Thus $c \approx 1$ when $\Delta_{0}<\lambda_{*}$ so in that case our consideration can be restricted to the two lowest energy levels.

Consider the adiabatic dielectric susceptibility at different temperatures. For high temperature $T>\lambda_{*}$ the quadrupole interaction is not significant and we may use the result of the standard tunneling model

$$
\chi_{T L S}(T) \approx\left(P \mu^{2} / 3\right) \ln (W / T), \quad \lambda_{*}<T<W .
$$

Now consider the limit of low temperatures, where the main contribution to the temperature dependence comes from the TLS's with modified tunneling splitting Eq. (6). According to Eq. (3), the TLS contribution to the dielectric constant can be estimated by the logarithmic integral with the lower limit $\Delta_{0, m i n}$, defined by the condition $E_{\text {min }}\left(\Delta_{0, \text { min }}\right)=T$. At low temperature this minimum energy splitting is given by the renormalized tunneling amplitude $\Delta_{0 *}=\eta_{*} \Delta_{0}$ (see Eq. (6) ) and the integral in Eq. (3) should be cut-off at $\Delta_{0, \min }=T / \eta_{*}$ rather than at $\Delta_{0, \min }=T$ as in the standard tunneling model. This leads to a negative correction to Eq. (10) of the form

$$
\chi_{T L S}(T) \approx\left(P \mu^{2} / 3\right)\left(\ln (W / T)-\ln \left(1 / \eta_{*}\right) .\right.
$$

The condition for the applicability of Eq. (11) is given by the criterion Eq. (17) which has to be valid for tunneling system with the smallest involved tunneling amplitudes, in particular $\Delta_{0, \text { min }}<\lambda_{*}$. Using $\Delta_{0, \min }<T / \eta_{*}$ we obtain the criterion for the validity of Eq. (11)

$$
T<\eta_{*} \lambda_{*} .
$$

The value of Eq. (11) at the highest admissible temperature $\eta_{*} \lambda_{*}$ coincides with the value of Eq. (10) at the lowest admissible temperature $\lambda_{*}$. Since the adiabatic dielectric constant cannot decrease with decreasing temperature, because excited states have a negative effect on it, we expects that it does not change at intermediate temperatures (plateau regime)

$$
\chi_{T L S}(T) \approx\left(P \mu^{2} / 3\right) \ln \left(W / \lambda_{*}\right), \quad \lambda_{*} \eta_{*}<T<\lambda_{*} .
$$

The plateau can also be interpreted as the consequence of the pseudogap in the distribution of effective tunneling amplitudes $\Delta_{0 *}$ within the range $\lambda_{*} \eta_{*}<\Delta_{0 *}<\lambda_{*}[16$.

To compare the theory with the experiment let us estimate the parameter $\lambda_{*}$. We can use the approximate expression obtained above, i.e., $\lambda_{*} \simeq n b$ where $n$ is the number of atoms per two level system and the energy $b$ has been defined before. The majority of glasses showing the plateau in the temperature dependent dielectric constant [5, 6, 7] contains $K, N a, A l, B a$ atoms which possess a relatively large nuclear quadrupole moment (see 13, 16]). The nuclear quadrupole splitting energy $b$ measured for these nuclei in different environments varies between $0.4 m K$ and $1 m K\left[16\right.$. To obtain $\lambda_{*} \sim 2 n b \sim 5 m K$ (see Eq. (7)) one can suppose that $n \approx 4$ atoms are involved in the tunneling motion. This estimate looks quite reasonable taking into account the strong effect of each tunneling atom on its neighboring atoms. Tunneling of the single atom shifts equilibrium positions of its neighbors. During the tunneling event the neighbors move to new equilibrium positions simultaneously with the tunneling atom. Therefore they participate in tunneling and should be included into the definition of the parameter $n$, which can thus become larger than 1 . The overlap parameter $\eta_{*}=<l g \mid r g>$ is taken between the ground states of $n$ non-interacting nuclear spins and estimated as

$$
\eta_{*}=\eta^{n} .
$$

where $\eta$ is the ground state overlap integral for one spin.

For a crude estimate of $\eta$ we consider the orientational glass $(K B r)_{1-x}(K C N)_{x}$ [17] where the $C N$ group rotates between different equilibrium positions by an angle $\phi=\cos ^{-1}(1 / 3) \approx 0.123[\underline{3}]$. For the sake of simplicity consider the simplified model of a nuclear spin $I=1$ with a quadrupole interaction described by the axially symmetric Hamiltonian [10] $b\left(I_{\alpha}^{2}-2 / 3\right)$ and $b>0$. The index $\alpha$ denotes the nuclear quadrupole axis. Then 


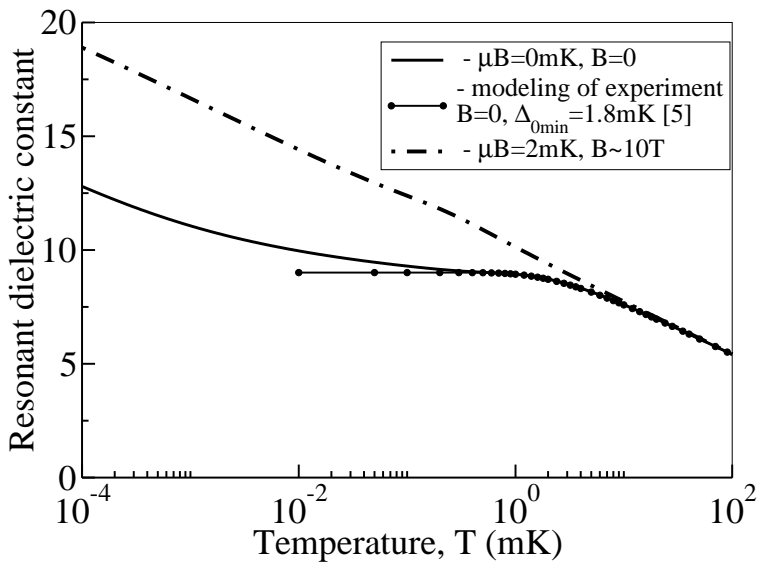

FIG. 1: Temperature and field dependence of TLS dielectric constant. The solid line shows the temperature dependence of the dielectric constant, calculated using parameters $\lambda_{*}=4 m K, \eta=0.33, n=4$ and the tunneling amplitude $\Delta_{0}$ replaced with $\Delta_{0 *}=\Delta_{0} \eta^{\left.n\left(1-\left(\Delta_{0} / \lambda_{*}\right)^{2}\right)^{3 / 2}\right)}$ at $\Delta_{0}<\lambda_{*}$ as derived in Ref. [16]. The artificially generated solid line with circles represents experimental data [5], which can be described within the standard tunneling model with the tunneling amplitude cutoff $\Delta_{0 \mathrm{~min}}=1.8 \mathrm{mK}$ (representative value, see [5]). It is clear that our approach does not deviate from the experiment in the experimental temperature range $T>1 \mathrm{mK}$. Dielectric constant in the external magnetic field (dot-dashed line) is found using the numerical modeling for axially symmetric nuclear quadrupole interaction [16].

one may estimate a single nuclear overlap integral as $\eta \approx \cos (\phi) \approx 0.33$. If a TLS consists of $n=4$ tunneling atoms, the effective overlap integral for two ground states can be estimated as $\eta_{*} \approx 10^{-2}$. In this case $\eta_{*} \lambda_{*} \approx 50 \mu \mathrm{K}$ and the plateau should show up in the temperature range from $T_{\text {down }} \sim 50 \mu K$ to $T_{\text {up }} \sim 4 m K$ (see Eq. (13)). For $T<50 \mu K$ the logarithmic temperature dependence of the dielectric constant should be restored and resemble the one at high temperatures (see Fig. 11). Note that the measurements of $T_{\text {down }}$ and $T_{u p}$ ) will permit us to estimate the number of atoms participating in TLS as $n \sim \ln \left(T_{\text {up }} / T_{\text {down }}\right)$. This is important for understanding the microscopic nature of tunneling systems.

The theory can be verified by the measurements of the dielectric constant at low temperatures $T<\lambda_{*}$ under the strong magnetic field, affecting the nuclear magnetic moments. When the field strength becomes comparable to the nuclear quadrupole interaction, it changes the spin quantization axes in both left and right wells aligning the nuclear spins along the field. Accordingly the mismatch of spin quantization axes is reduced by the magnetic field and the overlap integral $\eta_{*}$ increases and approaches unity in the large field limit. Thus we predict that a very strong magnetic field eliminates the influence of the nuclear quadrupole interaction on the dielectric constant and restore the standard tunneling model behavior. The critical magnetic field strength at which the nuclear quadrupole effects are eliminated can be estimated by requiring that the Zeeman splitting in the field is comparable with the energy $\lambda_{*} \sim 4 m K$. This expectation agrees with our numerical estimate [16]. Assuming that for the typical nuclear magnetic moment $\mu$ one has $\mu B \sim 1 \mathrm{mK}$ at $B \sim 5 T$, one can estimate the magnetic field necessary to eliminate the plateau in the dielectric constant to be $B \simeq 10 T$. The calculations of the temperature dependence of the dielectric constant at zero and large $B$ [16] are shown in Fig. 10 together with the plot, representing the experimental data [5].

In summary, we explained the plateau in the temperature dependence of the dielectric constant by the localization of the tunnelling particle by nuclear quadrupole interactions. We predict that an external magnetic field $B>10 T$ will restore the logarithmic temperature dependence of the dielectric constant in the whole temperature range. Such an experiment will verify the theory.

$\mathrm{AB}$ and YS are supported by the Louisiana Board of Regents (Contract LEQSF (2005-08)-RD-A-29). IYP is supported by the program of Russian Scientific school.

[1] P.W. Anderson, B.I. Halperin, C.M. Varma, Philos. Mag. 25, 1 (1972)

[2] W.A.Phillips, J.Low Temp.Phys. 7, 351 (1972).

[3] S. Hunklinger, A. K. Raychaudchary, Progr. Low Temp. Phys. 9, 267 (1986).

[4] W. A. Phillips, Rep. Prog. Phys. 50, 1567 (1987).

[5] S. Rogge, D. Natelson, B. Tigner, D. D. Osheroff, Phys. Rev. B 55, 11256 (1997).

[6] A. L. Burin, D. Natelson, D. D. Osheroff, Yu. Kagan, in "Tunneling Systems in Amorphous and Crystalline Solids", ed. P. Esquinazi, p. 243 ( Springer, 1998)

[7] P. Strehlow, W. Wolhardt, A. G. M. Jansen, R. Haueisen, G. Weiss, C. Enss, S. Hunklinger, Phys. Rev. Lett. 84, 1938 (2000).

[8] C. Enss, Physica B 316, p. 12 (2002).

[9] D. J. Salvino, S. Rogge, B. Tigner, D. D. Osheroff, Phys. Rev. Lett. 73, 268 (1994).

[10] A. Würger, A. Fleischmann, C. Enss, Phys. Rev. lett. 89, 237601 (2002).

[11] D. Bodea, A. Würger, J. Low Temp. Phys. 136, 59 (2004)

[12] I. Ya. Polishchuk, P. Fulde, A.L. Burin, Yu. Sereda, D. Balamurugan, J. Low Temp. Phys. 140, 355 (2005)

[13] To our knowledge the only one glass shows no plateau in the dielectric constant, which is mylar, containing no nuclei with spin $I>1 / 2$ [5]. This experimental result and similar results in other systems support our theory [16].

[14] P. Nagel, A. Fleischmann, S. Hunklinger, C. Enss, Phys. Rev. Lett. 92, 245511 (2004).

[15] Nuclear spin Hamiltonians for $n$-atomic tunneling system can be represented as the sum of $n$ single atom quadrupole interactions [16]. For integer nuclear spin the ground state is non-degenerate. The same is true for the whole Hamiltonian. If the nuclear spin is half-integer, it has a double-degenerate eigenstate and the ground state of $n$ spins is $2^{n}$ times degenerate. One can show [16] that these ground states can be separated into independent $2^{n}$ pairs, which can be treated as a non-degenerate ones.

[16] A. L. Burin, I. Ya Polishchuk, P. Fulde, Yu. Sereda, submitted to Phys. Rev. B; cond-mat/0509810 
[17] J. N. Dobbs, M. C. Foote, A. C. Anderson, Phys. Rev. B 33, 4178 (1986). 